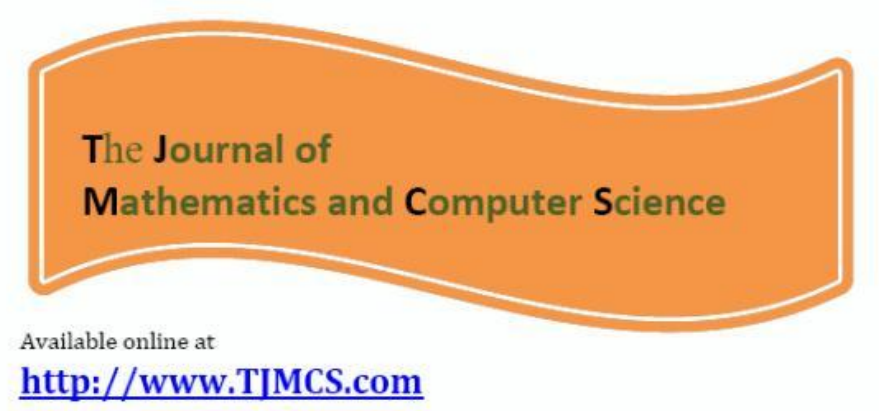

The Journal of Mathematics and Computer Science Vol .2 No.3 (2011) 495-514

\title{
A COMPILATION OF SOME WELL-KNOWN RESULTS IN RENORMING THEORY
}

\author{
Hadi Haghshenas ${ }^{a, 0}$, Saber Ghasempour ${ }^{b}$ \\ ${ }^{a}$ Department of Mathematical Analysis, Birjand University, Birjand, Iran \\ ${ }^{b}$ Faculty of Basic Science, Payamnoor University of Mazandaran, Amol, Iran \\ Received 27 July 2010; accepted 30 November 2010
}

Available online 19 January 2011

\begin{abstract}
The problems concerning equivalent norms of Banach spaces lie at the heart of Banach space theory. Our aim in this paper is to present a history of the subject, and to introduce some open problems.
\end{abstract}

\section{INTRODUCTION.}

Banach space theory is a classic topic in functional analysis. The study of the structure of Banach spaces provides a framework for many branches of mathematics

\footnotetext{
${ }^{0}$ Corresponding author.

E-mail addresses: haghshenas60@gmail.com (H. Haghshenas), ghasempour_pnu@yahoo.com (S. Ghasempour).
}

2010 Mathematics Subject Classification: 46B20, 46B03.

Keywords and Phrases: Gateaux differentiable norm, Fréchet differentiable norm, smooth norm, strictly convex space, uniformly convex space, Kadec-Klee property, Asplund space, weaklycompactly-generated space, Vasak space, Mazur intersection property, isomorphically polyhedral space, Schauder basis, uniform Eberlein compact space, super reflexive space.

This paper is based on a part of the first author's M.Sc thesis written under the supervision of professor Aman A. Assadi in Birjand university of Iran. 


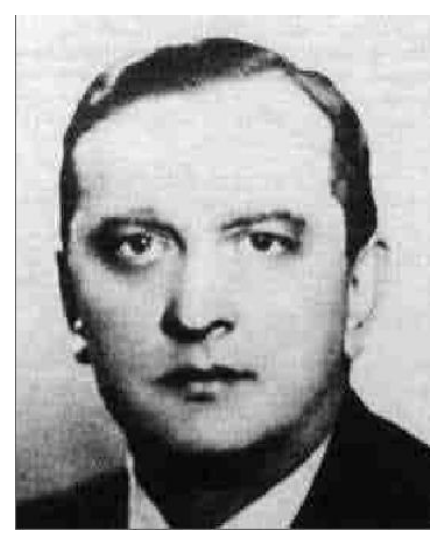

FIGURE 1. Stephan Banach 1892-1945

like differential calculus, linear and nonlinear analysis, abstract analysis, topology, probability, harmonic analysis, etc. The geometry of Banach spaces plays an important role in Banach space theory. Since it is easier to do analysis on a Banach space which has a norm with good geometric properties than on a general space, we consider in this survey an area of Banach space theory known as renorming theory. Renorming theory is involved with problems concerning the construction of equivalent norms on a Banach space with nice geometrical properties of convexity or differentiability. An excellent monograph containing the main advances on renorming theory until 1993 is [14].

Definition 1.1. Two norms |.| and $\|$.$\| on a Banach space X$ are called equivalent if there exist positive constants $A, B$ with $A\|x\| \leq|x| \leq B\|x\|$ for all $x \in X$.

If $X$ is finite dimensional all norms on $X$ are equivalent.

Through this paper $X$ is a real Banach space with norm $\|$.$\| and dual X^{*}, S(X)$ and $B(X)$ are, respectively, the unit sphere and the closed unit ball of $X$. In addition, $\ell^{\infty}, \ell^{p}$ denote the space of all real sequence $\left(x_{n}\right)_{n=1}^{\infty}$ such that $\left(x_{n}\right)_{n=1}^{\infty}$ is bounded, and $\sum_{n=1}^{\infty}\left|x_{n}\right|^{p}<\infty$, respectively. The reader is referred to [23] for undefined terms and notation. 


\section{Differentiable nORMS AND RENORMing THEORY.}

Differentiability of functions on Banach spaces is a natural extension of the notion of a directional derivative on $\mathbb{R}^{n}$. A function $f: X \rightarrow \mathbb{R}$ is said to be Gateaux differentiable at $x \in X$ if there exists a functional $A \in X^{*}$ such that $A(y)=\lim _{t \rightarrow 0} \frac{f(x+t y)-f(x)}{t}$, for all $y \in X$. In this case, $A$ is called Gateaux derivative of $f$. If the above limit exists uniformly for each $y \in S(X)$, then $f$ is called Fréchet differentiable at $x$ with Fréchet derivative $A$. Similarly, the norm $\|$.$\| of X$ is Gateaux or Fréchet differentiable at a non-zero $x \in X$ if the function $f(x)=\|x\|$ has the same property at $x$. Two important more strong notions of differentiability are obtained as uniform versions of both Fréchet and Gateaux differentiability. The norm $\|$.$\| of X$ is uniformly Fréchet differentiable if $\lim _{t \rightarrow 0} \frac{\|x+t y\|-\|x\|}{t}$ exists uniformly for $(x, y) \in S(X) \times S(X)$. Also, it is uniformly Gateaux differentiable if for each $y \in S(X), \lim _{t \rightarrow 0} \frac{\|x+t y\|-\|x\|}{t}$ exists uniformly in $x \in S(X)$.

In the general, Gateaux differentiability not imply Fréchet differentiability. In this case, we have the following result of $\mathrm{R}$. R. Phelps which provides a norm on $\ell^{1}$ that is Gateaux differentiable at all $x \neq 0$, but nowhere Fréchet differentiable. We follow [49], and refer the reader to this excellent book for the details. If $\|\cdot\|_{\infty}$ is the canonical supremum norm on $\ell^{\infty}$, define norm on $\ell^{\infty}$ by

$$
\|x\|_{*}=\|x\|_{\infty}+\left(\sum_{i=1}^{\infty} 2^{-i} x_{i}^{2}\right)^{\frac{1}{2}} .
$$

Then it is established in [49] that $\|\cdot\|_{*}$ is a dual norm on $\ell^{\infty}=\left(\ell^{1}\right)^{*}$, and its predual norm $\|$.$\| on \ell^{1}$ satisfies the necessary requirements.

Most of our attention will be concentrated on the differentiability of the norm function.

Lemma 2.1. The norm $\|$.$\| of X$ is Fréchet differentiable at $0 \neq x \in X$ if and only if $\lim _{t \rightarrow 0} \frac{\|x+t y\|+\|x-t y\|-2\|x\|}{t}=0$, uniformly for each $y \in S(X)$. 
Around the year of 1940, S̆mulian proved his following fundamental dual characterization of differentiability of norms, which is utilized in many basic renorming results.

Theorem 2.2. [23] For given Banach space $(X,\|\cdot\|)$ with dual space $\left(X^{*},\|\cdot\|_{*}\right)$ and $x \in S(X)$, the following are equivalent:

(i). $\|$.$\| is Fréchet differentiable at x$.

(ii). For all $\left(f_{n}\right)_{n=1}^{\infty},\left(g_{n}\right)_{n=1}^{\infty} \subseteq S\left(X^{*}\right)$, if $\lim _{n \rightarrow \infty} f_{n}(x)=1$ and $\lim _{n \rightarrow \infty} g_{n}(x)=1$, then $\lim _{n \rightarrow \infty}\left\|f_{n}-g_{n}\right\|_{*}=0$.

(iii). Each $\left(f_{n}\right)_{n=1}^{\infty} \subseteq S\left(X^{*}\right)$ with $\lim _{n \rightarrow \infty} f_{n}(x)=1$ is convergent in $S\left(X^{*}\right)$.

Proof. $\mathbf{i} \Rightarrow$ ii) Since the norm of $X$ is Fréchet differentiable at $x$, by the lemma 2.1., for each $\varepsilon>0$ there exists $\delta>0$ such that

$$
\|x+y\|+\|x-y\| \leq 2+\varepsilon\|y\|
$$

for any $y$ with $\|y\|<\delta$. Suppose $\left(f_{n}\right)_{n=1}^{\infty},\left(g_{n}\right)_{n=1}^{\infty} \subseteq S\left(X^{*}\right)$ with $\lim _{n \rightarrow \infty} f_{n}(x)=\lim _{n \rightarrow \infty} g_{n}(x)=1$. Since $\lim _{n \rightarrow \infty} f_{n}(x)=1$, there exists $N_{1} \in \mathbb{N}$ such that $\left|f_{n}(x)-1\right|<\varepsilon \delta$ for each $n \geq N_{1}$. Similarly, there is $N_{2} \in \mathbb{N}$ such that $\left|g_{n}(x)-1\right|<\varepsilon \delta$ for each $n \geq N_{2}$. Let $M=\max \left\{N_{1}, N_{2}\right\}$. Then for any $n>M$

$$
\left|f_{n}(x)-1\right|<\varepsilon \delta \text { and }\left|g_{n}(x)-1\right|<\varepsilon \delta,
$$

and we have

$$
\begin{aligned}
\left(f_{n}-g_{n}\right)(y) & =f_{n}(x+y)+g_{n}(x-y)-f_{n}(x)-g_{n}(x) \\
& \leq\|x+y\|+\|x-y\|-f_{n}(x)-g_{n}(x) \\
& \leq 2+\varepsilon\|y\|-f_{n}(x)-g_{n}(x) \\
& \leq\left|f_{n}(x)-1\right|+\left|g_{n}(x)-1\right|+\varepsilon\|y\| \\
& \leq 3 \varepsilon \delta .
\end{aligned}
$$


Hence for each $n>M$

$$
\left\|f_{n}-g_{n}\right\|_{*}=\sup _{h \in S(X)}\left(f_{n}-g_{n}\right)(h)=\sup _{h \in S(X)} \frac{\left(f_{n}-g_{n}\right)(\delta h)}{\delta} \leq 3 \varepsilon
$$

ii $\Rightarrow \mathbf{i}$ ) Suppose norm is not Fréchet differentiable at $x$. By the lemma 2.1., there is $\varepsilon>0$ such that for each $\delta=\frac{1}{n},\left\|x+y_{n}\right\|+\left\|x-y_{n}\right\|>2+\varepsilon\left\|y_{n}\right\|$ for some $y_{n}$ with $\left\|y_{n}\right\| \leq \frac{1}{n}$. Now by a version of Hahn-Banach theorem, for each $n \in \mathbb{N}$, there are $\left(f_{n}\right)_{n=1}^{\infty},\left(g_{n}\right)_{n=1}^{\infty} \subseteq S\left(X^{*}\right)$ such that

$$
f_{n}\left(x+y_{n}\right)=\left\|x+y_{n}\right\| \quad \text { and } \quad g_{n}\left(x-y_{n}\right)=\left\|x-y_{n}\right\| .
$$

Also for each $n \in \mathbb{N}$

$$
\left|\left\|x+y_{n}\right\|-\|x\|\right| \leq\left\|y_{n}\right\| \leq \frac{1}{n} .
$$

Thus $\left|f_{n}\left(x+y_{n}\right)-1\right|<\frac{1}{n}$ which implies that $\lim _{n \rightarrow \infty} f_{n}\left(x+y_{n}\right)=1$. On the other hand, $\left|f_{n}\left(y_{n}\right)\right| \leq\left\|y_{n}\right\| \leq \frac{1}{n}$ which follows that $\lim _{n \rightarrow \infty} f_{n}\left(y_{n}\right)=0$. Therefore $\lim _{n \rightarrow \infty} f_{n}(x)=1$. In a similar way, $\lim _{n \rightarrow \infty} g_{n}(x)=1$. Since

$$
g_{n}(x) \leq\left\|g_{n}\right\|_{*}=1 \quad \text { and } \quad f_{n}(x) \leq\left\|f_{n}\right\|_{*}=1
$$

we have $f_{n}(x)+g_{n}(x) \leq 2$. Thus we conclude that

$$
\left(f_{n}-g_{n}\right)\left(y_{n}\right) \geq\left\|x+y_{n}\right\|+\left\|x-y_{n}\right\|-2>\varepsilon\left\|y_{n}\right\| .
$$

Consequently $\left\|f_{n}-g_{n}\right\|_{*} \geq \varepsilon$ which is a contradiction.

ii $\Rightarrow$ iii) Let $\left(f_{n}\right)_{n=1}^{\infty} \subseteq S\left(X^{*}\right)$ such that $\lim _{n \rightarrow \infty} f_{n}(x)=1$. There is some $f \in S\left(X^{*}\right)$ such that $f(x)=\|x\|=1$. For each $n \in \mathbb{N}$, let $g_{n}=f$. Then $\lim _{n \rightarrow \infty} g_{n}(x)=f(x)=1$. Hence using ii we find that $\lim _{n \rightarrow \infty}\left\|f_{n}-g_{n}\right\|_{*}=0$ which means that $\left(f_{n}\right)_{n=1}^{\infty}$ is convergent.

iii $\Rightarrow$ ii) Consider $\left(f_{n}\right)_{n=1}^{\infty},\left(g_{n}\right)_{n=1}^{\infty} \subseteq S\left(X^{*}\right)$ with

$$
\lim _{n \rightarrow \infty} f_{n}(x)=\lim _{n \rightarrow \infty} g_{n}(x)=1 .
$$


For each $y \in X$, define $h_{n}(y)=f_{\frac{n+1}{2}}(y)$ for odd values of $n$ and $h_{n}(y)=g_{\frac{n}{2}}(y)$ for even values of $n$. So we have

$$
h_{2 n}=g_{n} \in S\left(X^{*}\right) \quad \text { and } \quad h_{2 n-1}=f_{n} \in S\left(X^{*}\right) \text {, }
$$

and therefore $h_{n} \in S\left(X^{*}\right)$. Moreover

$$
h_{2 n}(x)=g_{n}(x) \rightarrow 1 \quad \text { and } \quad h_{2 n-1}(x)=f_{n}(x) \rightarrow 1 .
$$

Hence $h_{n}(x) \rightarrow 1$. Using iii there is $h \in S\left(X^{*}\right)$ such that $\left\|h_{n}-h\right\|_{*} \rightarrow 0$. Then

$$
\left\|f_{n}-g_{n}\right\|_{*}=\left\|h_{2 n-1}-h_{2 n}\right\|_{*} \leq\left\|h_{2 n-1}-h\right\|_{*}+\left\|h_{2 n}-h\right\|_{*} \rightarrow 0,
$$

as required.

The separable and reflexive Banach spaces occupy an important role in our investigations, because they contain numerous nice structural aspects.

Recall that $X$ is reflexive if a certain natural isometry of $X$ into $X^{* *}$ is onto. This mapping is ${ }^{\wedge}: X \rightarrow X^{* *}$ giving by $\widehat{x}\left(x^{*}\right)=x^{*}(x)$.

As a direct application of S̆mulian's theorem, we have the following corollary:

Corollary 2.3. [23] If the dual norm of $X^{*}$ is Fréchet differentiable then $X$ is reflexive.

Proof. A standard theorem in functional analysis courses is that $X$ is reflexive if and only if each nonzero $f \in X^{*}$ attains its norm at some $x \in S(X)$. Let $f \in S\left(X^{*}\right)$ and choose $\left(x_{n}\right)_{n=1}^{\infty} \in S(X)$ such that $f\left(x_{n}\right) \rightarrow 1$. By the S̆mulian's theorem, $\lim _{n \rightarrow \infty} x_{n}=x \in S(X)$. Therefore

$$
f(x)=f\left(\lim _{n \rightarrow \infty} x_{n}\right)=\lim _{n \rightarrow \infty} f\left(x_{n}\right)=1=\|f\|_{*} .
$$

If now $f \in X^{*}$ is non-zero, then $\frac{f}{\|f\|_{*}} \in S\left(X^{*}\right)$ and according to the above manner there exists $x \in S(X)$ such that $\frac{f}{\|f\|_{*}}(x)=1$.

Theorem 2.4. [23] The following assertions imply the reflexivity of $X$.

(i). The norm of $X$ is uniformly Fréchet differentiable.

(ii). The third dual norm of $X$ is Gateaux differentiable (Giles-Kadec-Phelps). 
Theorem 2.5. [23] The following statements imply separability of the dual space of a separable Banach space $X$.

(i). The second dual norm of $X$ is Gateaux differentiable.

(ii). The norm of $X$ is Fréchet differentiable (Kadec-Restrepo).

Theorem 2.6. [26] If $X$ is reflexive then can be renormed in such a way that both $X$ and $X^{*}$ have Fréchet differentiable norm.

There exist reflexive spaces which do not admit an uniformly Gateaux differentiable norm. This example can be found in Kutzarova and Troyanski [38]. However, we do have the following positive result.

Theorem 2.7. $[11,59]$ If $X$ is separable, then $X$ admits an uniformly Gateaux differentiable norm

Theorem 2.8. (Kadec) If $X^{*}$ is separable then $X$ admits an equivalent Fréchet differentiable norm.

As a consequence we obtain (combining theorems 2.8. and 2.5.(i)):

Corollary 2.9. A separable Banach space $X$ which has Gateaux differentiable second dual norm, admits an equivalent Fréchet differentiable norm.

\section{ASPLUND SPACES AND RENORMING THEORY.}

If every continuous, convex function $f$ defined on an open and convex subset $C$ of $X$ is Fréchet differentiable on a dense $G_{\delta}$ subset of $C$, we say that $X$ is Asplund. Recall that the dual of a separable Banach space need not to be separable in general.

Theorem 3.1. $[17,40]$ Let $X$ be a separable Banach space. Then the following are equivalent:

(i). $X^{*}$ is separable.

(ii). $X$ is Asplund.

(iii). $X$ admits an equivalent Fréchet differentiable norm.

(iv). $X$ does not admit an equivalent rough norm. ( A norm $\|\cdot\|$ of $X$ is rough if 
there exists $\varepsilon>0$ such that for all $x \in X, \limsup _{\|y\| \rightarrow 0} \frac{\|x+y\|+\|x-y\|-2\|x\|}{\|y\|} \geq \varepsilon$ )

\section{Strictly CONVEX SPACES AND EQUiVAlEnt NORMS.}

One interesting and fruitful line of research, dating from the early days of Banach space theory, has been to relate analytic properties of a Banach space to various geometric conditions on that space. The simplest example of such a condition is that of strict convexity.

The space $(X,\|\|$.$) (or the norm \|$.$\| of X$ ) is called strictly convex (R) if for $x, y \in$ $S(X),\|x+y\|=2$ implies $x=y$, in other words, when $S(X)$ does not contain non-trivial segments.

For example, the Banach space $\mathbb{R}^{2}$ with Euclidean norm $\|(x, y)\|_{2}=\sqrt{x^{2}+y^{2}}$ is strictly convex.

Recall that the space $c_{0}:=\left\{\left(x_{n}\right)_{n=1}^{\infty}: x_{n} \in \mathbb{R}, \lim _{n \rightarrow \infty} x_{n}=0\right\}$ is a Banach space when endowed with the canonical norm $\left\|\left(x_{n}\right)\right\|_{\infty}=\max _{n \in \mathbb{N}}\left\{\left|x_{n}\right|\right\}$. Since for the first two standard basis vectors $e_{1}$ and $e_{2}$ of $c_{0}$ we have $\left\|e_{1}\right\|_{\infty}=\left\|e_{2}\right\|_{\infty}=\frac{\left\|e_{1}+e_{2}\right\|_{\infty}}{2}$, it follows that $c_{0}$ is not strictly convex with this norm.

Strict convexity is not preserved by equivalent norms. For example consider the norm

$$
\||(x, y)|\|=\max \left\{2|x|,\|(x, y)\|_{2}\right\},
$$

on $\mathbb{R}^{2}$. Clearly, $\|||$.$\| is equivalent to the strictly convex Euclidean norm \|\cdot\|_{2}$. But $\||| \cdot||$ is not strictly convex, for if we take $(1,0),(1,1) \in \mathbb{R}^{2}$, then

$$
\||(1,0)|||=2,\||(1,1)| \|=2,
$$

while

$$
\||(1,0)+(1,1)|\|=\||(2,1)|\|=4 .
$$

There are few results devoted to strictly convex renormings, most of them are based on the following simple observation. Let $Y$ be a strictly convex Banach space and 
$T: X \rightarrow Y$ a linear one-to-one bounded operator; then $\||x|\|=\|x\|+\|T(x)\|$, $x \in X$, is an equivalent strictly convex norm on $X$.

Theorem 4.1. $[35,36]$ If $X$ is separable then $X^{*}$ admits an equivalent dual strictly convex norm.

Proof. Let $\|\cdot\|_{*}$ be a given dual norm on $X^{*}$ and $\left\{x_{i}\right\}_{i=1}^{\infty}$ be dense in $S(X)$. Define a norm $\||\cdot|\|$ on $X^{*}$ by $|\|f\||^{2}=\|f\|_{*}^{2}+\sum_{i=1}^{\infty} 2^{-i} f^{2}\left(x_{i}\right)$. Now one can show that $\||\cdot|\|$ is an equivalent dual norm on $X^{*}$ which is strictly convex. $\square$

\section{EQUiVAlENT NORMS AND SMOOTH NORMS.}

One of the most beautiful areas of Banach space theory is the close knit relationship between various notions of smoothness and convexity.

The norm $\|$.$\| of X$ is smooth at $x \in X \backslash\{0\}$ if there is a unique $f \in X^{*}$ such that $\|f\|_{*}=1$ and $f(x)=\|x\|$. We say that the norm $\|\cdot\|$ of $X$ (or $X$ for short) is smooth if $\|$.$\| is smooth at all x \in X \backslash\{0\}$. If the norm $\|$.$\| of X$ is smooth at non-zero $x \in X$, then $x$ is said to be a smooth point. A smooth point $x$ is said to be a preserved smooth point if the bidual norm is also smooth at $x$.

There exists an interest relation between differentiability and smoothness, as following.

Theorem 5.1. The norm of $X$ is smooth at $x \in X \backslash\{0\}$ if and only if it is Gateaux differentiable at $x$.

Regarding Gateaux smoothness, we have the following result.

Theorem 5.2. [23] If the dual norm on $X^{*}$ is strictly convex (Gateaux differentiable) then the norm of $X$ is Gateaux differentiable (strictly convex).

The converse implications in the above theorem are true for reflexive spaces, but not in general. Here we mention an example of Troyanski [54] which uses ordinal spaces. For $\omega_{1}$ the first uncountable ordinal, denote the collection of continuous functions 
on $\left[0, \omega_{1}\right]$ by $C\left(\left[0, \omega_{1}\right]\right)$. Then $C\left(\left[0, \omega_{1}\right]\right) \times \ell^{1}$ is a Banach space with Gateaux differentiable norm, the dual of which admits no dual strictly convex norm.

Example 5.3 The spaces $\ell^{p}, 1<p<\infty$, are strictly convex and smooth, while the spaces $\ell^{1}$ and $\ell^{\infty}$ are neither strictly convex nor smooth.

Theorem 5.4. [28] A separable Banach space is reflexive if and only if each smooth point is preserved in each equivalent norm.

A point $x \in C$ is called an extreme point of a convex subset $C$ of $X$ if $C \backslash\{x\}$ is convex, too.

The next theorem is the dual version of the above theorem:

Theorem 5.5. [29] A Banach space is reflexive if and only if for each equivalent norm the extreme points of the unit ball are preserved.

\section{RENORMING THEORY AND KADEC-KLEE PROPERTY.}

The norm $\|$.$\| of X$ has weak-Kadec-Klee property if $\left(x_{n}\right)_{n=1}^{\infty} \subseteq X$ converges weakly to some $x \in X$ and $\lim _{n \rightarrow \infty}\left\|x_{n}\right\|=\|x\|$, then $\lim _{n \rightarrow \infty}\left\|x_{n}-x\right\|=0$. Also, a dual norm $\|\cdot\|_{*}$ of $X^{*}$ has weak*-Kadec-Klee property if $\lim _{n \rightarrow \infty}\left\|f_{n}-f\right\|_{*}=0$, whenever $\left(f_{n}\right)_{n=1}^{\infty} \subseteq X^{*}$ is weak $k^{*}$-converges to some $f \in X^{*}$ and $\lim _{n \rightarrow \infty}\left\|f_{n}\right\|_{*}=\|f\|_{*}$.

The Kadec-Klee norms play an absolutely key role in geometric Banach space theory and its applications.

Theorem 6.1. [23] For each separable Banach space $X$, if the dual norm of $X^{*}$ has the $w e a k^{*}$-Kadec-Klee property, then $X^{*}$ is separable.

Combining above theorem with theorem 2.8., we have the following corollary:

Corollary 6.2. If $X$ is separable and the dual norm of $X^{*}$ has the weak*-KadecKlee property, then $X$ admits an equivalent Fréchet differentiable norm.

\section{LOCALLY UNIFORMLY CONVEX SPACES AND EQUIVALENT NORMS.}

The notion of locally uniformly convex norm was introduced by Lovaglia in [42]. The Banach space $(X,\|$.$\| ) (or the norm \|$.$\| of X$ ) is said to be locally uniformly 
convex (LUR) if

$$
\lim _{n \rightarrow \infty}\left(2\|x\|^{2}+2\left\|x_{n}\right\|^{2}-\left\|x+x_{n}\right\|^{2}\right)=0 \quad \Rightarrow \quad \lim _{n \rightarrow \infty}\left\|x-x_{n}\right\|=0,
$$

for any sequence $\left(x_{n}\right)_{n=1}^{\infty}$ and $x$ in $X$.

Lovaglia showed, as a straightforward consequence of theorem 2.2., that the norm of a Banach space is Fréchet differentiable if the dual norm is LUR. The converse do not hold, even up to renormings. In fact, there exists a space with a Fréchet differentiable norm, which does not admit any equivalent norm with a strictly convex dual norm [14]. However, in the class of spaces with unconditional bases, we do have equivalence up to a renorming.

Theorem 7.1. [33] If $X^{*}$ has a dual LUR norm then $X$ admit an equivalent LUR norm.

Theorem 7.2. [45] A Banach space $X$ with a Fréchet differentiable norm which has Gateaux differentiable dual norm admits an equivalent LUR norm.

Theorem 7.3. [35] If $X$ is separable, then $X$ admits an equivalent LUR norm. Moreover, if $X^{*}$ is separable then $X^{*}$ admits an equivalent dual norm that is LUR. Proof. We show the second statement. Let $\|\cdot\|_{*}$ be a given dual norm on $X^{*}$, $\left\{x_{i}\right\}_{i=1}^{\infty}$ be dense in $S(X)$ and $\left\{f_{i}\right\}_{i=1}^{\infty}$ be dense in $S\left(X^{*}\right)$. For $i \in \mathbb{N}$, put $F_{i}=$ $\operatorname{span}\left\{f_{1}, f_{2}, \ldots, f_{i}\right\}$. Define a norm $|\|\cdot\||$ on $X^{*}$ by

$$
\|\| f\left\|\left.\right|^{2}=\right\| f \|_{*}^{2}+\sum_{i=1}^{\infty} 2^{-i} \operatorname{dist}\left(f, F_{i}\right)^{2}+\sum_{i=1}^{\infty} 2^{-i} f^{2}\left(x_{i}\right)
$$

Then $|\|\|$.$| is an equivalent LUR norm on X^{*}$.

The above theorem shows that, in particular, every separable Banach space admits an equivalent strictly convex norm.

Another classical and powerful result of Troyanski [55] asserts that a Banach space $X$ admits an equivalent LUR norm if and only if it admits an equivalent weakKadec-Klee norm and an equivalent strictly convex norm. 


\section{Renorming theory And Uniformly CONVEX SPACES.}

The space $(X,\|\|$.$) (or the norm \|$.$\| of X$ ) is said to be uniformly convex (UR) if for all sequences $\left(x_{n}\right)_{n=1}^{\infty},\left(y_{n}\right)_{n=1}^{\infty} \subseteq X$

$$
\lim _{n \rightarrow \infty}\left(2\left\|x_{n}\right\|^{2}+2\left\|y_{n}\right\|^{2}-\left\|x_{n}+y_{n}\right\|^{2}\right)=0 \quad \Rightarrow \quad \lim _{n \rightarrow \infty}\left\|x_{n}-y_{n}\right\|=0 .
$$

It is not hard to show that any Hilbert space is uniformly convex.

We have $(\mathrm{UR}) \Rightarrow(\mathrm{LUR}) \Rightarrow(\mathrm{R})$, but the converse is not true, necessarily. For example, define a norm $|\|\cdot\||$ on $C[0,1]$ by $\mid\|f\|^{2}=\|f\|_{\infty}^{2}+\|f\|_{2}^{2}$, where $\|\cdot\|_{\infty}$ denotes the standard supremum norm of $C[0,1]$ and $\|\cdot\|_{2}$ denotes the canonical norm of $L^{2}[0,1]$, the $L^{2}$-space over the Lebesgue interval $[0,1]$. Then $|\|\cdot\|| \mid$ is strictly convex but not LUR on $C[0,1]$. Consider functions $f \equiv 1$ and $g_{n}$ for every $n$, where $g_{n}$ is the broken line determined by the points $(0,1),\left(\frac{1}{n}, 1\right),(1,1)$. It is easy to verify that $f, g_{n}$ fail to have the property of local uniform convexity [23].

There is a complete duality between uniform convexity and uniform Fréchet differentiability.

Theorem 8.1. ( $\breve{S} m u l i a n)$ For any Banach space $X$ with dual space $X^{*}$, the dual norm on $X^{*}$ is uniformly convex if and only if the norm of $X$ is uniformly Fréchet differentiable. Also, the dual norm on $X^{*}$ is uniformly Fréchet differentiable if and only if the norm of $X$ is uniformly convex.

The above theorem implies that the norm of every Hilbert space is uniformly Fréchet differentiable.

One of the first theorems to relate the geometry of the norm to linear topological properties is the following;

Theorem 8.2. (Milman-Pettis) Any uniformly convex Banach space is reflexive.

\section{MAZUR INTERSECTION PROPERTY AND EQUIVALENT NORMS.}

Recall that the space $X$ is said to have the Mazur intersection property (MIP) if every bounded closed convex set in $X$ is an intersection of closed balls. Equivalently, 
for any bounded closed convex set $K$ in $X$ and for any $x \notin K$, there exists a ball $B$, $B \supseteq K$ and $x \notin B$. S. Mazur was the first to study Banach spaces with MIP. R. R. Phelps [48] showed that for a finite-dimensional Banach space $X$ to have the MIP, it is necessary and sufficient that the set of extreme points of $B\left(X^{*}\right)$ is dense in $S\left(X^{*}\right)$.

Theorem 9.1. [41] $X$ has the MIP if and only if for any two disjoint bounded weak*-closed convex sets $K_{1}, K_{2}$ in $X^{* *}$, there exist balls $B_{1}^{* *}, B_{2}^{* *}$ in $X^{* *}$ with centers in $X$ such that $B_{i}^{* *} \supseteq K_{i}, i=1,2$, and $B_{1}^{* *} \cap B_{2}^{* *}=\emptyset$.

In 1978, J. Giles, D. Gregory and B. Sims [27] raised the question whether every Banach space with the MIP is an Asplund space. In 1995, M. J. sevilla and J. P. Moreno [51] has exhibited a class of non-Asplund spaces that admit equivalent norms with the MIP.

\section{BASIS AND RENORMING THEORY.}

A Hamel basis $\left(e_{\alpha}\right)$ for $X$ is a set of linearly independent vectors in $X$ such that each $x \in X$ is uniquely representable as a finite linear combination of $e_{\alpha}$. A Hamel basis for an infinite-dimensional Banach space must, in fact, be uncountable.

A Schauder basis for $X$ is a sequence $\left(x_{n}\right)_{n=1}^{\infty}$ of vectors in $X$ such that every vector in $X$ has a unique representation of the form $\sum_{n=1}^{\infty} \alpha_{n} x_{n}$ with each $\alpha_{n}$ a scalar and where the sum is converges in the norm topology.

A Schauder basis $\left(x_{n}\right)_{n=1}^{\infty}$ is said to be an unconditional basis provided that every permutation of $\left(x_{n}\right)_{n=1}^{\infty}$ is also a Schauder basis.

Theorem 10.1. [23] Every Banach space with a countable Hamel basis admits an equivalent Fréchet differentiable norm.

Theorem 10.2. [52] Let $X$ have an unconditional basis. Then $X$ admits an equivalent norm with an LUR dual norm whenever $X$ admits an equivalent Fréchet differentiable norm. 


\section{Equivalent NORMS AND WEAKLY-COMPACTLY-GENERATED SPACES.}

A wide class of Banach spaces, which still enjoy many useful topological properties, have proved to be a deciding generalization over the past forty years. They are defined as follows.

The Banach space $X$ is said to be weakly-compactly-generated (WCG) if there exists a weakly compact set $K \subseteq X$ whose closed linear span is $X$.

Recall that the space $X$ is separable if there exists a countable set $\left\{x_{n}\right\}_{n=1}^{\infty}$ with $\overline{\left\{x_{n}\right\}_{n=1}^{\infty}}=X$. An important characterization of reflexivity is the result that $X$ is reflexive if and only if $B(X)$ is weakly compact.

Remark. If $X$ is reflexive, then one may take $K=B(X)$ in the above definition, whereas if $X$ is separable, with $\left\{x_{n}\right\}_{n=1}^{\infty}$ dense in the $S(X)$, we can take $K=\left\{n^{-1} x_{n}\right\}_{n=1}^{\infty} \bigcup\{0\}$. In this way we see that both separable and reflexive spaces are (WCG).

Theorem 11.1. [53] Every (WCG) space admits an equivalent LUR norm.

The above theorem shows that every reflexive Banach space admits an equivalent Fréchet differentiable norm with weak-Kadec-Klee property. Also, we obtain that if $X^{*}$ is a (WCG) space, $X$ admits an equivalent Fréchet differentiable norm.

Theorem 11.2. (Amir-Lindenstrauss) If $X$ is a (WCG) space, $X^{*}$ admits an equivalent strictly convex dual norm.

Theorem 11.3. [5] If $X$ is a (WCG) and Asplund space, $X^{*}$ admits an equivalent LUR norm.

\section{VASAK SPACES AND EQUIVALENT NORMS.}

A class of spaces wider than (WCG) spaces, known as weakly countably determined or Vasak spaces, was originally defined and investigated by Vasak.

The space $X$ is Vasak if there is a sequence $\left(B_{n}\right)_{n=1}^{\infty}$ of weak $k^{*}$-compact sets in $X^{* *}$ such that given $x \in X$ and $u \in X^{* *} \backslash X$, there is $n \in \mathbb{N}$ such that $x \in B_{n}$ and $u \notin B_{n}$. 
Theorem 12.1. [26] If $X^{*}$ is Vasak then $X$ admits an equivalent Fréchet differentiable norm.

Theorem 12.2. [43] Every Vasak space has an equivalent norm the dual of which is strictly convex.

Many of the renorming results for (WCG) Banach spaces actually apply to Vasak spaces. Further details can be found in [19].

\section{UNIFORM EBERLEIN COMPACT SPACES AND RENORMING THEORY.}

Recall that a compact space $K$ is called a uniform Eberlein compact if $K$ is homeomorphic to a weakly compact subset of a Hilbert space in its weak topology.

Theorem 13.1. (Fabian-Godefroy-Zizler) [22] $\left(B\left(X^{*}\right), w^{*}\right)$ is uniform Eberlein compact if and only if $X$ admits an equivalent uniformly Gateaux differentiable norm.

\section{SUPER-REFleXivity AND RENORMING THEORY.}

Given Banach spaces $X, Y$ and $\varepsilon>0$, we say that $Y$ is finitely representable in $X$ if for every finite-dimensional subspace $Z$ of $Y$, there is an isomorphism $T$ of $Z$ onto $T(Z) \subseteq X$ such that $\|T\|\left\|T^{-1}\right\|<1+\varepsilon$. A Banach space $X$ is said to be super-reflexive if every Banach space finitely representable in $X$ is reflexive.

It is not hard to show that every super-reflexive Banach space, is reflexive.

One of the well-known super-reflexive Banach spaces are Hilbert spaces. One may think that these are the only examples of super-reflexive spaces. In the following theorem, we see that this is far from the case, indeed the family of super-reflexive Banach spaces is quite a rich one.

Theorem 14.1. (Enflo-James) $X$ is super-reflexive if and only if $X$ admits an equivalent uniformly Fréchet differentiable norm.

In [50] Pisier gave a new proof, based on probabilistic methods with martingales, of a theorem of Enflo [18]: every super-reflexive Banach space admits an equivalent uniformly convex norm. Inspired by the martingale approach of Pisier, in 1979 
Troyanski stated the first characterization of existence of LUR renormings in Banach spaces [53].

\section{EqUiVAlENT NORMS AND ISOMORPHICALLY POLYHEDRAL SPACES}

Recall that a set $B \subseteq X^{*}$ is called a boundary for $X$ if for every $x \in X$ there is a $f \in B$ such that $f(x)=\|x\|$.

The norm of $X$ is said to be polyhedral if the unit ball of every finite-dimensional subspace of $X$ has finitely many extreme points. The Banach space $X$ is isomorphically polyhedral if there is an equivalent polyhedral norm on $X$. Isomorphically polyhedral spaces were studied first by Fonf. He proved that a separable isomorphically polyhedral Banach space has a separable dual.

Theorem 15.1. [15] A separable Banach space $X$ is isomorphically polyhedral if and only if $X$ admits an equivalent norm with a countable boundary.

Therefore, if the separable Banach space $X$ admits an equivalent norm with a countable boundary, then $X$ is isomorphically polyhedral and consequently, $X$ admits a Fréchet differentiable equivalent norm.

\section{Some interesting PROBlems.}

The following problems in this area arise:

(Q1) If the Banach space $X$ has the Radon-Nykodym property (i.e., every bounded closed convex subset of $X$ is the closed convex hull of its strongly exposed points.), does it follow that $X$ admits an equivalent weak-Kadec-Klee norm? Does it admits an equivalent strictly convex norm?

(Q2) Does every Asplund space admit an equivalent SSD norm? Recall that the norm $\|$.$\| on X$ is called strongly subdifferentiale (SSD) if for each $x \in X$, the onesided limit $\lim _{t \rightarrow 0^{+}} \frac{\|x+t y\|-\|x\|}{t}$ exists uniformly on $y$ in $S(X)$.

(Q3) Assume that a Banach space $X$ admits an equivalent Gateaux differentiable norm and that $X$ admits also an equivalent SSD norm. Does $X$ admit an equivalent 
Fréchet differentiable norm?

(Q4) Assume that $X$ is a nonseparable non Asplund space. Does $X$ admit an equivalent norm that is nowhere SSD except at the origin? For separable non Asplund space the answer is yes.

(Q5) Assume that the norm of a separable Banach space $X$ has the property that its restriction to every infinite dimensional closed subspace $Y \subseteq X$ has a point of Fréchet differentiability on $Y$. Is then $X^{*}$ necessarily separable?

(Q6) Assume that $X$ is Vasak. Does $X$ admit an equivalent norm that has the following property: $\left(f_{n}\right)_{n=1}^{\infty}$ is weak ${ }^{*}$-convergent to some $f \in B\left(X^{*}\right)$ whenever $f_{n} \in S\left(X^{*}\right)$ are such that $\left\|f_{n}+f_{m}\right\| \rightarrow 2$ as $n, m \rightarrow \infty$ ?

(Q7) Assume that $X$ has an unconditional basis and admits an equivalent Gateaux differentiable norm. Does $X$ admit an equivalent norm the dual of which is strictly convex?

\section{REFERENCES}

[1] D. Amir and J. Lindenstrauss, The structure of weakly compact sets in Banach spaces, Ann. of Math. 88 (1968), 35-46.

[2] E. Asplund, Frechet differentiability of convex functions, Acta Math. 121 (1968), 31-47.

[3] Y. Benyamini and J. Lindenstrauss, Geometric nonlinear functional analysis, American Math. Society, 2000.

[4] E. Bishop and R. R. Phelps, A proof that every Banach space is subreflexive, Bull. Amer. Math. Soc. 67 (1961), 97-98.

[5] J. M. Borwein and J. D. Vanderwerff, A survey on renorming and set convergence, Topological Methods in Nonlinear Analysis, 5 (1995), 211-228.

[6] J. M. Borwein and D. Preiss, A smooth variational principle with applications to subdifferentiability and to differentiability of convex functions, Trans. Amer. Math. Soc. 303 (1987), $517-527$.

[7] I. Cioranescu, Geometry of Banach spaces, duality mappings and nonlinear problems, Vol 62, Kluwer Academic Publishers, Dordrecht, Paises Bojos, 1990.

[8] J. Clarkson, Uniformly convex spaces, Trans. Amer. Math. Soc. 40 (3) (1936), 396-414. 
[9] M. M. Day, Strict convexity and smoothness of normed spaces, Trans. Amer. Math. Soc. 78 (1955), 516-528.

[10] M. M. Day, Uniform convexity in factor and conjugate spaces, Ann. of Math. 45 (1944), 375-385.

[11] M. M. Day, R. C. James and S. Swaminathan, Normed linear spaces that are uniformly convex in every direction, Can. J. of Math. 23 (1972), 1051-1059.

[12] R. Deville, V. Fonf and P. Hajek, Analytic and ployhedral approximation of convex bodies in separable Banach spaces, Israel J. of Math. 105 (1998), 139-154.

[13] R. Deville, G. Godefroy and V. Zizler, Smooth bump functions and geometry of Banach spaces, Matematika 40 (2) (1993), 305-321.

[14] R. Deville, G. Godefroy and V. Zizler, Smoothness and renormings in Banach spaces, Pitman Monographs and Surveys in Pure and Appl. Math. 64 (1993).

[15] J. Diestel, Geometry of Banach spaces. Selected Topics. Lecture Notes in Math. SpringerVerlag, 1975.

[16] S. S. Dragomir, Inner product inequalities for two equivalent norms and applications, Acta Math. Vietnam, 34 (3) (2009), 361-369.

[17] I. Ekeland and G. Lebourg, Generic Frechet differentiability and perturbed optimization problems in Banach spaces, Trans. Amer. Math. Soc. 224 (1976), 193-216.

[18] P. Enflo, Banach spaces which can be given an equivalent uniformly convex norm, Israel J. of Math. 13 (1972), 281-288.

[19] M. Fabian, Gateaux differentiability of convex functions and topology, Canadian Math. Soc. Series of Monographs and Advanced Texts, 1997.

[20] M. Fabian, Each weakly countably determined Asplund space admits a Frechet smooth norm, Bull. Austr. Math. Soc. 36 (1987), 367-374.

[21] M. Fabian et al., Hilbert-generated spaces, J. Func. Anal. 200 (2003), 301-323.

[22] M. Fabian et al., Smoothness in Banach spaces. selected problems. Rev. R. Acad. Cien. Serie. A. Mat. 100 (2006), 101-125.

[23] M. Fabian et al., Functional analysis and infinite-dimensional geometry, Springer-Verlag, New York, 2001.

[24] M. Fabian et al., Uniform eberlein compacta and uniformly Gateaux smooth norms, Serdica Math. J. 23 (3-4) (1997), 351-362.

[25] M. Fabian et al., Norms with locally Lipschitzian derivatives, Israel J. of Math. 44 (1983), 262-276. 
[26] R. Fry and S. McManus, Smooth bump functions and the geometry of Banach spaces, Expo. Math. 20 (2002), 143-183.

[27] J. Giles, D. A. Gregory and B. Sims, Characterization of normed linear spaces with Mazur's intersection property, Bull. Austral. Math. Soc. 18 (1978), 105-123.

[28] B. V. Godun, Points of smoothness of convex bodies in separable Banach spaces, Matem. Zametki, 38 (1985), 713-716.

[29] B. V. Godun, Preserved extreme points, Functional. Anal. i Prilozhen., 19 (1985), 75-76.

[30] A. S. Granero et al., Intersections of closed balls and geometry of Banach spaces, Extracta Math. 1 (19) (2004), 55-92.

[31] P. Hajec, Dual renormings of Banach spaces, Comment Math. Univ. Carol. 37 (1996), 241253.

[32] P. Hajec and M. Johanis, Characterization of reflexivity by equivalent renorming, J. Func. Anal. 211 (2004), 163-172.

[33] R. Haydon, The three space problem for strictly convex renormings and the quotient problem for Frechet smooth renormings, Seminaire Initiation Analyse, Universite Paris VI (1992-1993).

[34] R. C. James, Super reflexive spaces with bases, Pacific J. of Math. 41 (1972), 409-419.

[35] M. I. Kadec, Spaces isomorphic to locally uniformly rotund spaces, Izv. Vyss. Uc. Zav. Matem. 1 (1959), 51-57 and $\mathbf{1}$ (1961), 186-187.

[36] M. I. Kadec, Conditions on differentiability of the norm of a Banach space, Uspekhi Mat. Nauk. 20 (1965), 183-187.

[37] A. Kriegl and P. W. Michor, The convenient setting of global Analysis, Math. Surveys and Monographs, 1997.

[38] D. Kutzarova and S. Troyanski, Reflexive Banach spaces without equivalent norms which are uniformly convex or uniformly differentiable in every direction, Studia Math. 72 (1982), 91-95.

[39] S. Lajara and A. J. Pallares, Renorming and operators, Extracta Math. 19 (1) (2004), 141144 .

[40] E. B. Leach and J. H. M. Whitfield, Differentiable functions and rough norms on Banach spaces, Proc. Math. Amer. Soc. 33 (1972), 120-126.

[41] B.-L. Lin, Ball separation properties in Banach spaces and extermal properties of unit ball in duall spaces, Taiwanese J. Math. 1 (4) (1997), 405-416.

[42] A. R. Lovaglia, Locally uniformly convex Banach spaces, Trans. Amer. Math. Soc. 78 (1955), $225-238$. 
[43] S. Mercourakis, On weakly countably determined Banach spaces, Trans. Amer. Math. Soc., 300 (1987), 307-327.

[44] A. Molto, J. Orihuela, S. Troyanski and V. Zizler, Strictly convex renormings, J. London Math. Soc. 75 (2007), 647-658.

[45] A. Molto, J. Orihuela, S. Troyanski and M. Valdivia, A nonlinear transfer technique for renorming, Lecture Notes in Math., Springer, New York.

[46] A. Molto, J. Orihuela, S. Troyanski and M. Valdivia, On weakly locally uniformly rotund Banach spaces, J. of func. Anal. 163 (1999), 252-271.

[47] I. Namioka and R. R. Phelps, Banach spaces which are Asplund spaces, Duke Math. J. 42 (1975), 735-750.

[48] R. R. Phelps, A representation theorem for bounded closed convex sets, Proc. Amer. Math. Soc. 102 (1960), 976-983.

[49] R. R. Phelps, Convex functions, monotone operators and differentiability, Second Edition. Lecture Notes in Mathematics, 1364 Springer-Verlag, Berlin, 1993.

[50] G. Pisier, Martingales with values in uniformly convex spaces, Israel J. Math. 20 (3-4) (1975), 326-350.

[51] M. J. Sevilla and J. P. Moreno, The Mazur intersection property and Asplund spaces, C. R. Acad. Sci. Paris 321 (1995), 1219-1223.

[52] R. J. Smith and S. L. Troyanski, Unconditional bases and strictly convex dual renormings, Bull. London Math. Soc. 41 (2009), 831-840.

[53] S. L. Troyanski, On equivalent local uniformly convex norms, C. R. Acad. Bulgare Sci. 32 (9) (1979), 1167-1169

[54] S. L. Troyanski, An example of smooth space whose dual is not strictly convex, (Russian) Studia Math. 35 (1970), 305-309.

[55] S. L. Troyanski, On a property of the norm which is close to local uniformly rotundity, Math. Ann. 271 (2) (1985), 305-313.

[56] J. Vanderwerff, Smooth approximations in Banach spaces, Proc. Amer. Math. Soc. 115 (1992), 113-120.

[57] J. Wells, Differentiable functions on Banach spaces with Lipschitz derivatives, J. Diff. Geom. 8 (1973), 135-152.

[58] D. Yost, Asplund spaces for beginners, Acta. Univ. Carolin. Math. Phys. 34 (1993), 159-177.

[59] V. Zizler, On some rotundity and smoothness properties of Banach spaces, Dissertationnes Math. (Rozprawy Mat.) 87 (1971), 1-33. 